\title{
Head-to-head comparison of dipyridamole, dobutamine and pacing stress echocardiography for the detection of myocardial ischemia in an animal model of coronary artery stenosis
}

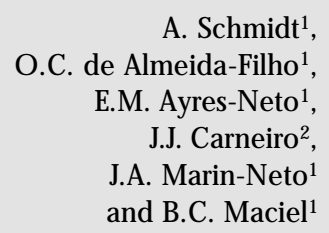

A. Schmidt ${ }^{1}$,

O.C. de Almeida-Filho ${ }^{1}$, E.M. Ayres-Neto ${ }^{1}$,

J.J. Carneiro ${ }^{2}$,

J.A. Marin-N eto ${ }^{1}$ and B.C. Maciel ${ }^{1}$

\author{
1Divisão de Cardiologia and 2D epartamento de Cirurgia, \\ Hospital das Clínicas, Faculdade de Medicina de Ribeirão Preto, \\ Universidade de São Paulo, Ribeirão Preto, SP, Brasil
}

\section{Correspondence \\ A. Schmidt \\ Divisão de Cardiologia \\ HC, FMRP, USP \\ 14049-900 Ribeirão Preto, SP \\ Brasil \\ Fax: + 55-16-633-0869 \\ E-mail: aschmidt@usp.br}

Publication supported by FAPESP

Received October 16, 2000 Accepted April 2, 2001

\section{Abstract}

To compare the sensitivity of dipyridamole, dobutamine and pacing stress echocardiography for the detection of myocardial ischemia we produced a physiologically significant stenosis in the left circumflex artery of 14 open-chest dogs (range: 50 to $89 \%$ reduction in luminal diameter). In each study, dobutamine (5 to $40 \mu \mathrm{g} \mathrm{kg}^{-1} \mathrm{~min}^{-1}$ in 3-min stages) and pacing ( $20 \mathrm{bpm}$ increments, each $2 \mathrm{~min}$, up to $260 \mathrm{bpm}$ ) were performed randomly, and then followed by dipyridamole (up to $0.84 \mathrm{mg} /$ $\mathrm{kg}$ over $10 \mathrm{~min})$. The positivity of stress echocardiography tests was quantitatively determined by a significant $(\mathrm{P}<0.05)$ reduction of or failure to increase absolute and percent systolic wall thickening in the stenotic artery supplied wall, as compared to the opposite wall (areas related to the left anterior descending artery). Systolic and diastolic frozen images were analyzed off-line by two blinded observers in the control and stress conditions. The results showed that 1) the sensitivity of dobutamine, dipyridamole and pacing stress tests was 57,57 and $36 \%$, respectively; 2) in animals with positive tests, the mean percent change of wall thickening in left ventricular ischemic segments was larger in the pacing $(-19 \pm 11 \%)$ and dipyridamole $(-18 \pm 16 \%)$ tests as compared to dobutamine $(-9 \pm 6 \%)(\mathrm{P}=0.05)$, but a similar mean reduction of wall thickening was observed when this variable was normalized to a control left ventricular segment (area related to the left anterior descending artery) (pacing: $-16 \pm 7 \%$; dipyridamole: $-25 \pm 16 \%$; dobutamine: $-26 \pm$ $10 \%$; not significant), and 3) a significant correlation was observed between magnitude of coronary stenosis and left ventricular segmental dysfunction induced by ischemia in dogs submitted to positive stress tests. We conclude that the dobutamine and dipyridamole stress tests showed identical sensitivities for the detection of myocardial ischemia in this one-vessel disease animal model with a wide range of left circumflex artery stenosis. The pacing stress test was less sensitive, but the difference was not statistically significant. The magnitude of segmental left ventricular dysfunction induced by ischemia was similar in all stress tests evaluated.
Key words

- Stress echocardiography

- Pacing

- Dobutamine

- Dipyridamole

- Animal model 


\section{Introduction}

Stress echocardiography has become a clinically useful method for detection, prognosis and therapeutic decisions in patients with known or suspected coronary heart disease. Dipyridamole, dobutamine and atrial pacing are stress modalities commonly used in the clinical setting as an alternative to exercise. The documented accuracy of these stress echocardiographic techniques is quite variable. Reported sensitivity values for the detection of ischemia range from 40 to $92 \%, 68$ to $96 \%$ and 75 to $93 \%$, respectively, for dipyridamole (1-6), dobutamine (7-11) and pacing (12-19), while specificity ranges from 93 to $100 \%, 66$ to $100 \%$ and 76 to $100 \%$.

The reported accuracy of stress echocardiography to identify regional wall motion abnormalities induced by ischemia is influenced not only by the physician's ability to perform and interpret the echocardiographic images (20), but also by a number of clinical setting, anatomical and physiological factors including heterogeneity of the patients studied, variability of stress protocols, severity, location and extent of coronary disease, relative significance of diseased vessels, presence of collateral circulation, and degree of ventricular hypertrophy (16). As a consequence of this inherent diversity, it is quite difficult to determine from the clinical investigations reported which stress modality is most sensitive for the detection of myocardial ischemia.

Therefore, this investigation was designed to compare, in an animal model of one-vessel coronary heart disease with a wide range of physiologically significant coronary stenosis: 1) sensitivity of dipyridamole, dobutamine and pacing stress echocardiography for the detection of myocardial ischemia, and 2) the magnitude of segmental left ventricular dysfunction induced by ischemia.

\section{Material and Methods}

\section{Animal preparation}

Fourteen mongrel dogs of either sex ( 14.5 to $30 \mathrm{~kg}$, mean: $20.8 \mathrm{~kg}$ ) were included in this study. The experimental protocol agreed with the guidelines of the American Heart Association about the use of research animals (21). Dogs were anesthetized with intravenous pentobarbital sodium ( $33 \mathrm{mg} / \mathrm{kg}$ ), intubated and placed under mechanical ventilation with $100 \%$ oxygen (Takaoka, model $600)$ through an endotracheal tube. Anesthesia was maintained with additional doses of pentobarbital sodium when necessary.

Aortic and pulmonary pressure (phasic and mean) were continuously monitored by catheters connected to adequately calibrated fluid-filled transducers. The left femoral vein was cannulated for fluid and drug infusion. A lead II electrocardiogram was recorded continuously.

A median thoracotomy was performed and the heart was suspended in a pericardial cradle. A segment of the left circumflex artery was dissected and an adjustable mechanical occluder was positioned around the vessel. An epicardial pacemaker electrode was implanted in the right atrial appendage.

Coronary anatomy was documented by coronary angiography at baseline and then after producing variable degrees of coronary stenosis by adjusting the mechanical occluder. In each animal, a coronary constriction greater than $50 \%$ was produced based on visual estimation after repeated contrast injections. Quantitative coronary angiography analysis was performed off-line using a computerized system (ImageComm Quantim IC-Software). Reduction of luminal coronary diameter ranged from 50 to $89 \%$ (mean: $59 \pm 12 \%$ ), corresponding to an area reduction of 75 to $99 \%$ (mean: $89 \pm 7 \%$ ). Coronary stenosis that produced segmental wall motion abnormalities in the baseline echocardiogram was avoided by continuous monitor- 
ing. At the end of the experiment, the animals were sacrificed by the administration of potassium chloride following an additional injection of pentobarbital.

\section{Echocardiographic study}

Two-dimensional echocardiography was performed using a Hewlett-Packard (Sonos 1000; Andover, MA, USA) ultrasound system with a $5-\mathrm{MHz}$ transducer. Echocardiographic images were obtained with the transducer placed on the epicardial surface and the ultrasound beam oriented to provide short axis images at the midpapillary level. Wall motion was continuously monitored throughout the experiment and images were stored on $1 / 2$ inch VHS videotape for later analysis.

\section{Experimental protocol}

Each animal was submitted to all three modalities of stress during the same experimental session, i.e., pacing and dobutamine in a random order, and finally dipyridamole. After each stress modality, sufficient time was allowed for recovery of baseline conditions. A continuous infusion pump (model 933; Harvard Apparatus Co., Southnatick, MA, USA) was used for drug infusion. Atrial pacing was produced by a Medtronic generator (model 5325) starting with a rate 20 pulses per minute (ppm) higher than baseline heart rate, with $20 \mathrm{ppm}$ increases each 2 min up to a heart rate of $260 \mathrm{bpm}$, or up to the time when segmental wall motion abnormalities or atrioventricular conduction defects occurred. Intravenous dobutamine was started at a dose of $5 \mu \mathrm{g} \mathrm{kg}^{-1} \mathrm{~min}^{-1}$ and increased every 3 min to $10,20,30$ or $40 \mu \mathrm{g}$ $\mathrm{kg}^{-1} \mathrm{~min}^{-1}$. Infusion was interrupted if segmental wall motion abnormalities or severe arrhythmia occurred. Dipyridamole infusion was performed using the high dose protocol $(0.56 \mathrm{mg} / \mathrm{kg}$ over $4 \mathrm{~min}$, followed $4 \mathrm{~min}$ later by an additional $0.28 \mathrm{mg} / \mathrm{kg}$ over $2 \mathrm{~min}$ ). Drug administration was interrupted if wall motion abnormalities were documented. Echocardiographic images were recorded on videotape at baseline and at the final $30 \mathrm{~s}$ of each protocol step.

\section{Data analysis}

Heart rate, systolic arterial pressure and rate-pressure product were measured before and at the end of each stress test and are expressed as mean \pm standard deviation for three consecutive beats.

The end-diastolic and end-systolic frames selected for analysis were the one showing the largest and the smallest endocardial-limited cavity areas, respectively. The left ventricular end-diastolic area was measured by planimetry using a software incorporated into the ultrasound system and was expressed as the mean of three cardiac cycles. Left ventricular wall end-diastolic and end-systolic dimensions were measured in the area supplied by the circumflex coronary artery (risk region) and in the area supplied by the left anterior descending coronary artery (control region). Mean \pm standard deviation for seven cardiac cycles was obtained. Systolic wall thickening (systolic wall dimension diastolic wall dimension), expressed in centimeters, and percent systolic wall thickening $\{[($ systolic wall dimension - diastolic wall dimension)/diastolic wall dimension] $\mathrm{x}$ $100\}$ were calculated for both ventricular regions of interest. "Normalization" of wall thickening for the control region was calculated by the formula: change (stress - baseline) of percent systolic thickening in the risk area minus change (stress - baseline) of percent systolic thickening in the control area. Positivity of the stress echocardiography test was quantitatively determined by a significant $(\mathrm{P}<0.05)$ reduction of or failure to increase systolic wall thickening and percent systolic wall thickening in the circumflex as compared to the left anterior descending artery-related regions. Measurements were performed by two experienced observers who 
reached consensus on each measurement.

\section{Statistical analysis}

Data are reported as mean $\pm \mathrm{SD}$. Comparisons of baseline to peak stress values were made by analysis of variance for repeated measures. The paired or unpaired two-tailed Student $t$-test was used when appropriate. McNemar's test was used for comparison of sensitivity of the stress tests applied. Simple linear regression analysis was used to assess the correlation between coronary stenosis severity and the magnitude of segmental wall motion abnormalities induced by the various stress modalities. Probability was considered statistically significant at $\mathrm{P}<0.05$.

\section{Results}

The stress test was interrupted before the peak target of the experimental protocol as a consequence of 1) occurrence of A-V conduction disturbances in 13 animals during pacing, 2) ventricular (3 animals) or supraventricular arrhythmias (1 animal) induced by dobutamine, and 3) occurrence of

Figure 1. Heart rate, systolic blood pressure and rate-pressure product at baseline and at the end of each stress test. PAC, atrial pacing; $\mathrm{DOB}$, dobutamine infusion; DIP, dipyridamole infusion.

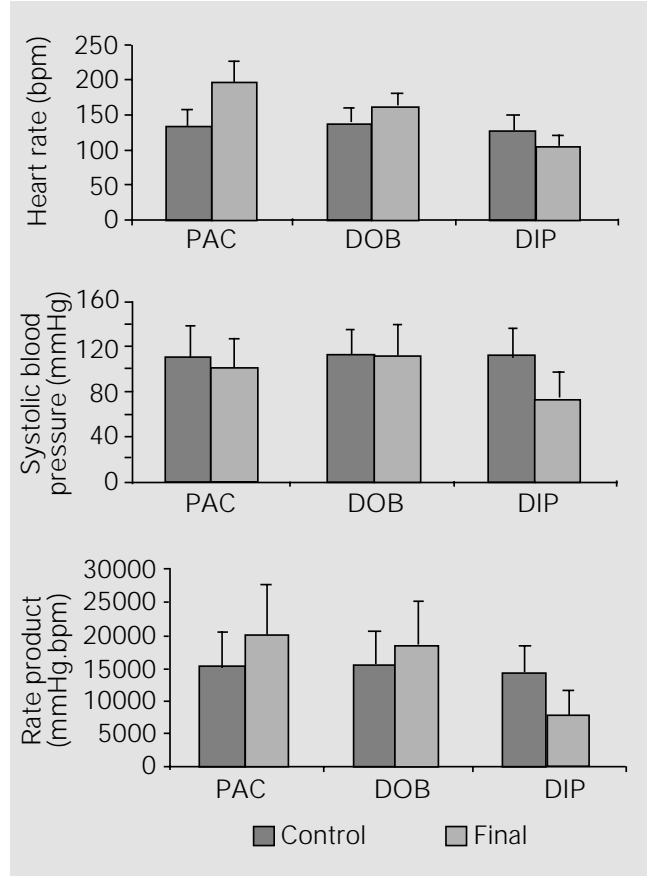

segmental wall motion abnormalities ( 2 animals) during dipyridamole administration.

\section{Hemodynamic data}

Mean baseline heart rate for pacing, dobutamine and dipyridamole was $134 \pm 23$, $135 \pm 26$ and $126 \pm 24 \mathrm{bpm}$, respectively, while systolic arterial pressure was $109 \pm 28$, $110 \pm 24$ and $112 \pm 25 \mathrm{mmHg}$. Mean values of rate-pressure product were $15083 \pm 5370$, $15221 \pm 5559$ and $14295 \pm 4106 \mathrm{mmHg}$.bpm, for pacing, dobutamine and dipyridamole, respectively. There was no significant difference in the three hemodynamic variables between the baseline states. Under stress conditions, heart rate increased to $194 \pm 34$ bpm for pacing $(\mathrm{P}<0.001 \mathrm{vs}$ baseline $)$ and to $158 \pm 23 \mathrm{bpm}(\mathrm{P}=0.002)$ for dobutamine and decreased to $101 \pm 19 \mathrm{bpm}(\mathrm{P}<0.001)$ with dipyridamole, while systolic arterial pressure was $100 \pm 28$ (no significant difference from baseline), $110 \pm 29$ (not significant) and $72 \pm 27 \mathrm{mmHg}(\mathrm{P}<0.001)$ for pacing, dobutamine and dipyridamole, respectively. The rate-pressure product reached $20022 \pm 7770(\mathrm{P}<0.001), 18032 \pm 7148(\mathrm{P}=$ $0.03)$ and $7586 \pm 4011 \mathrm{mmHg} . b p m(\mathrm{P}<0.001)$ during pacing, dobutamine and dipyridamole, respectively (Figure 1).

Baseline left ventricular end-diastolic area was not significantly different for the three modalities of stress. Before starting pacing, dobutamine and dipyridamole, left ventricular end-diastolic area was $5.24 \pm 2.31,5.64 \pm 2.55$ and $4.98 \pm 1.94 \mathrm{~cm}^{2}$, respectively. Pacing did not change end-diastolic area $(4.42 \pm 2.09$ $\mathrm{cm}^{2}$; no significant difference from baseline), dobutamine induced a significant reduction in area $\left(3.38 \pm 1.44 \mathrm{~cm}^{2}, \mathrm{P}=0.03\right)$, while dipyridamole infusion resulted in a small, nonsignificant increase in left ventricular diastolic dimension $\left(5.21 \pm 1.84 \mathrm{~cm}^{2}\right)$ (Figure 2).

\section{Sensitivity of stress test modalities}

In this investigation, the stress test was 
considered to present an ischemic response (positive test) in the presence of a significant $(\mathrm{P}<0.05)$ reduction of or failure to increase absolute systolic wall thickening and percent systolic wall thickening in the circumflex artery compared to the left anterior descending artery-related regions. Using this quantitative criterion, sensitivity was 36,57 and $57 \%$, for pacing, dobutamine and dipyridamole, respectively. The differences were not statistically significant.

\section{Magnitude of segmental wall motion abnormalities}

Mean baseline values of absolute systolic wall thickening and percent wall thickening were similar for all stress modalities, both in control and risk regions. Absolute systolic wall thickening in the left circumflex and anterior descending regions was $0.40 \pm 0.12$ and $0.40 \pm 0.11 \mathrm{~cm}$ before pacing, $0.45 \pm 0.16$ and $0.40 \pm 0.12 \mathrm{~cm}$ before dobutamine and $0.46 \pm 0.20$ and $0.39 \pm 0.09$ $\mathrm{cm}$ before dipyridamole, respectively. Percent systolic wall thickening was $35 \pm 10$ and $37 \pm 10 \%, 38 \pm 12$ and $36 \pm 9 \%, 39 \pm 14$ and $35 \pm 6 \%$, for pacing, dobutamine and dipyridamole, respectively.

In dogs that presented an ischemic response to the stress test, a significant reduction in percent systolic wall thickening was documented during pacing in the risk region (baseline: $40 \pm 15 \%$, pacing: $22 \pm 11 \% ; \mathrm{P}=$ 0.01 ), while in the left anterior descending region no significant change occurred (baseline: $34 \pm 8 \%$, pacing: $32 \pm 9 \%$ ). Dobutamine also reduced percent systolic wall thickening in the risk region in dogs with a positive stress test, but this change did not reach statistical significance (baseline: $37 \pm 10 \%$, dobutamine: $28 \pm 12 \%$ ). On the other hand, in the control region a significant increase of percent systolic wall thickening occurred (baseline: $41 \pm 13 \%$, dobutamine: $59 \pm 15 \%$; $\mathrm{P}=0.02$ ). A significant reduction of wall thickening was also documented during dypiridamole in the left circumflex region (baseline: $36 \pm 6 \%$, dipyridamole: $19 \pm 20 \%$; $\mathrm{P}=0.03$ ), while in the control region a small, nonsignificant increase in percent wall thickening (baseline: $38 \pm 10 \%$, dipyridamole: 45 $\pm 8 \%$ ) was observed.

Comparing the absolute variation of percent systolic wall thickening in the risk and control regions induced by the three stress modalities, more pronounced reduction occurred in systolic thickening during pacing $(-19 \pm 11 \%)$ and dipyridamole $(-18 \pm 16 \%)$ compared to dobutamine $(-9 \pm 6 \%)$. However, when the variation of systolic thickening was normalized to the control region, the magnitude of induced wall motion abnormality was not significantly different for the three modalities of stress (pacing: $-16 \pm 7 \%$, dobutamine: $-26 \pm 10 \%$, dipyridamole: $-25 \pm$ 16\%) (Figure 3).
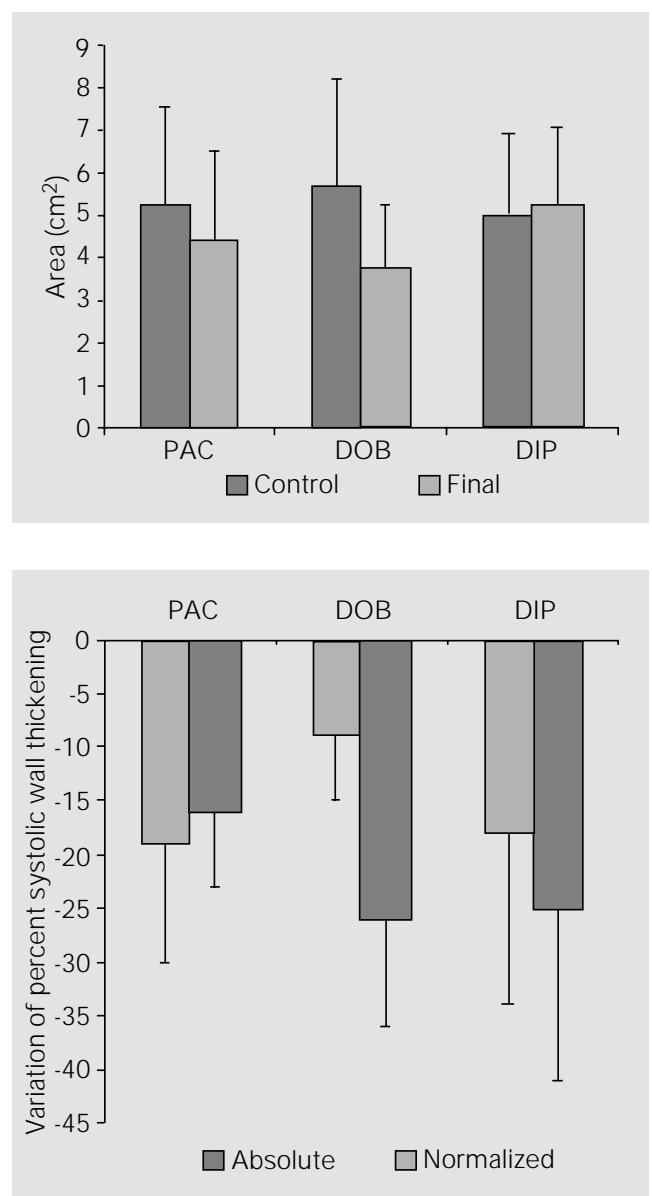

Figure 2. End-diastolic left ventricular area at baseline and at the end of each stress test. PAC, atrial pacing; $\mathrm{DOB}$, dobutamine infusion; DIP, dipyridamole infusion.

Figure 3. Absolute and normalized variation of percent systolic wall thickening in the risk (circumflex artery) and in control (left anterior descending artery) regions in animals with a positive stress test. PAC, atrial pacing; $\mathrm{DOB}$, dobutamine infusion; DIP, dipyridamole infusion. 


\section{Relationship between left ventricular dysfunction induced by ischemia and stenosis severity}

When positive tests for ischemia were considered, the correlation between the change of the relative systolic thickening in the risk region normalized to the control region and the severity of coronary stenosis, as evaluated by luminal diameter reduction, was statistically significant for dobutamine ( $\mathrm{r}=0.72, \mathrm{P}=0.046)$ but not for dipyridamole $(r=-0.45, P=0.26)$. However, a marked improvement in correlation was observed for dobutamine $(\mathrm{r}=0.84, \mathrm{P}=0.036)$ and dipyridamole $(\mathrm{r}=0.83, \mathrm{P}=0.043)$ when two dogs which had important left ventricular hypertrophy and coronary stenosis severity in the lower range were excluded from the analysis.

\section{Discussion}

\section{Characteristics of the experimental model}

The experimental model used in this investigation remained quite stable throughout the different phases of the study protocol as documented by the similar mean values for the hemodynamic variables measured (heart rate, systolic arterial pressure and ratepressure product) and by the mean diastolic area of the left ventricle before each test. These observations indicate that, although dipyridamole was always the last stress test applied, this sequence probably had no significant effect, by itself, on the results obtained.

Using an animal model with one-vessel coronary stenosis ranging from 50 to $89 \%$ of luminal diameter reduction we were able to simulate coronary heart disease in a range of stenosis considered significant in the clinical setting. The variable degree of coronary stenosis used in this animal model appears to be adequate for sensitivity and specificity studies, considering that a $50 \%$ reduction of the luminal diameter represents the best cut- off value for the functional significance of a stenosis (22). Moreover, conditions for comparing sensitivities of different stress tests appear to be more adequate when a moderate degree of coronary stenosis such as that used in this investigation (mean: $69 \pm 12 \%$ of luminal diameter reduction) is present.

\section{Sensitivity of the stress tests}

In this study, the stress test was considered to be positive for ischemia when it elicited a significant $(\mathrm{P}<0.05)$ reduction of or failure to increase systolic wall thickening and percent systolic wall thickening in the circumflex (stenosed) as compared to the left anterior descending artery (control)-related regions. This quantitative approach for positivity of the test was important to avoid subjective evaluation of wall motion during stress. In addition, the comparative analysis of wall motion in risk and control regions has been shown to be valuable for the detection of ischemia during stress echocardiography, considering that hyperkinesia may occur in the left ventricular region supplied by the non-stenosed coronary artery $(23,24)$.

The three tests did not differ significantly in sensitivity despite the higher value observed for dobutamine (57\%) and dipyridamole (57\%) as compared to the pacing stress test (36\%). Probably, the tendency to a lower sensitivity during pacing was related to the relatively large number of tests prematurely interrupted due to the occurrence of atrioventricular conduction disturbances.

To the best of our knowledge, there are no previous studies comparing sensitivity for detection of ischemia using simultaneously these three stress tests in the same experimental model as performed in this investigation. Fung et al. (25) reported sensitivity of $100 \%$ for dobutamine and of $55 \%$ for dipyridamole in an experimental dog model of circumflex coronary stenosis. However, two important differences between that investigation and the current one should be 
pointed out. In their model, only critical coronary stenosis was produced as opposed to the large range of coronary stenosis used in the present study. In addition, dipyridamole was infused up to a maximal dose of 0.56 $\mathrm{mg} / \mathrm{kg}$ body weight, while we used up to $0.84 \mathrm{mg} / \mathrm{kg}$ body weight in 12 out of 14 dogs. In the clinical setting, increased sensitivity has been documented when this larger dose of dipyridamole was used in stress echocardiography (26). Segar et al. (27) reported sensitivity of $100 \%$ for dobutamine and $75 \%$ for dipyridamole in a swine model of anterior descending coronary artery stenosis, but they also used only critical coronary stenosis. Therefore, under the conditions of the current investigation, dobutamine and dipyridamole seemed to be equally sensitive in producing systolic dysfunction secondary to myocardial ischemia, while atrial pacing showed a slightly lower, not significantly different, sensitivity.

There are no clinical investigations which directly compare these three stress tests in patients with one-vessel coronary disease. Some studies performed in groups of patients with one-vessel disease have shown slightly higher sensitivity for dobutamine (range: $50-76 \%$, mean: 66\%) than for dipyridamole (range: $31-82 \%$, mean: $52 \%$ ) (2832 ), while other studies have shown equivalent sensitivity for these stress tests $(33,34)$. Although a comparison of the current experimental study with available clinical data cannot be directly performed considering the large number of factors interfering with the response to stress in patients, it should be mentioned that the reported mean values of sensitivity are very close to those obtained in the present experimental study. On the other hand, the sensitivity we obtained for pacing $(36 \%)$ was much lower than that reported for patients with one-vessel disease (85\%) (17), probably as a consequence of the large number of pacing tests that were interrupted at an early stage due to atrioventricular conduction disturbances.

\section{Magnitude of segmental wall motion abnormalities}

Quantitative analysis of the magnitude of the segmental wall motion abnormalities induced by the stress tests showed that during pacing and dipyridamole the changes in percent systolic thickening in the risk region were comparable, but larger than those induced by dobutamine. However, when the variations in wall thickening were normalized for the control region supplied by the non-stenosed coronary artery, the three tests induced similar magnitudes of wall motion dysfunction. Our results are similar to those reported by Paulsen et al. (35) comparing exercise, pacing, dobutamine and dipyridamole in an experimental model. They demonstrated that pacing and exercise induced a more important wall motion abnormality, although the differences between all tests were reduced when the results were normalized to the non-ischemic region.

\section{Relationship between left ventricular dysfunction induced by ischemia and stenosis severity}

In the clinical setting, correlation between coronary stenosis severity and its functional consequences is not always good (36). A wide variety of factors contribute to the complex physiological consequences of coronary artery disease including severity, localization, extension and number of coronary lesions, presence of collateral circulation and ventricular hypertrophy, as well as dynamic changes of coronary tonus. On the other hand, a good correlation between these variables is usually obtained in experimental models $(37,38)$. In this study, a good correlation between the changes in percent systolic thickening in the risk region and the severity of coronary stenosis in animals with positive tests for ischemia during dobutamine and pacing was obtained only when two animals, with mild degree of stenosis and ventricular 
hypertrophy, were excluded from analysis. This observation suggests that in these two animals ventricular hypertrophy may have contributed to the occurrence of wall motion dysfunction during stress.

In this one-vessel disease animal model, the dobutamine and dipyridamole stress tests showed identical sensitivities for the detec- tion of myocardial ischemia in a wide range of left circumflex artery stenosis, while the pacing stress test tended to be less sensitive. On the other hand, the magnitude of segmental left ventricular dysfunction induced by ischemia was similar for all stress tests evaluated.

\section{References}

1. Picano E \& Lattanzi F (1991). Dipyridamole echocardiography. A new diagnostic window on coronary artery disease. Circulation, 83 (Suppl III): III.19-III.26.

2. Picano E, Lattanzi $F \&$ Masini M (1987). Comparison of high-dose dipyridamoleechocardiography test and exercise twodimensional echocardiography for diagnosis of coronary artery disease. American J ournal of Cardiology, 59: 539-542.

3. Margonato $A$, Chierchia $S \&$ Cianflone $D$ (1987). Limitations of dipyridamole-echocardiography in effort angina pectoris. American J ournal of Cardiology, 59: 225230.

4. Picano $\mathrm{E}$, Distante $\mathrm{A}$, Masini $\mathrm{M}$, Morales MA, Lattanzi F \& L'Abbate A (1985). Dipyridamole-echocardiography test in effort angina pectoris. American J ournal of Cardiology, 56: 452-456.

5. Previtali $M$, Lanzarini $L$, Ferrario $M$, Mussini A \& Montemartini C (1991). Dobutamine versus dipyridamole echocardiography in coronary artery disease. Circulation, 83 (Suppl III): III.27-III.31.

6. Mazeika P, Nihoyannopoulos P, J oshi J \& Oakley CM (1992). Uses and limitations of high dose dipyridamole stress echocardiography for evaluation of coronary artery disease. British Heart J ournal, 67: 144-149.

7. Berthe C, Pierard LA, Hiemaux M, Trotteur G, Lempereur P, CarlierJ \& Kulbertus HE (1986). Predicting the extent and location of coronary artery disease in acute myocardial infarction by echocardiography during dobutamine infusion. J ournal of the American College of Cardiology, 58: 1167-1172.

8. Sawada SG, Segar DS, Ryan T, Brown SE, Dohan AM, Williams R, Fineberg NS, Armstrong WF \& Feigenbaum H (1991). Echocardiography detection of coronary artery disease during dobutamine infusion. Circulation, 83: 1605-1614.

9. Sawada SG, Segar DS, Ryan T, Dohan
AM, Williams R \& Feigenbaum H (1992). Catecholamine stress echocardiography. Echocardiography, 9: 177-187.

10. Segar DS, Brown SE, Sada SG, Ryan T\& Feigenbaum H (1992). Dobutamine stress echocardiography: Correlation with coronary lesion severity as determined by quantitative angiography. J ournal of the American College of Cardiology, 19: 11971202.

11. Marwick T, D'Hondt A, Baudhuin $T$, Willemart B, Wijns W, Detry J M \& Melin J (1993). Optimal use of dobutamine stress for the detection and evaluation of coronary artery disease: Combination with echocardiography or scintigraphy, or both? J ournal of the American College of Cardiology, 22: 159-167.

12. Chapman PD, Doyle TP \& Troup PJ (1984). Stress echocardiography with transesophageal atrial pacing: Preliminary report of a new method for detection of ischemic wall motion abnormalities. Circulation, 70: 445-450.

13. Iliceto $S, D^{\prime} A m b r o s i o ~ G$, Sorino $M$, Papa A, Amico A, Ricci A \& Rizzon P (1985). Comparison of post exercise and transesophageal atrial pacing two-dimensional echocardiography for detection of coronary artery disease. Feasibility, specificity and sensitivity. American J ournal of Cardiology, 57: 547-553.

14. Iliceto $S$, Sorino $M \& D^{\prime}$ Ambrosio $G$ (1985). Detection of coronary artery disease by two-dimensional echocardiography and transesophageal atrial pacing. J ournal of the American College of Cardiology, 5: 1188-1197.

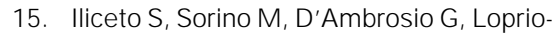
re V, Ricci A, Papa A, Amico A, Chiddo A \& Rizzon P (1986). Atrial pacing in the detection and evaluation of coronary artery disease. European Heart J ournal, 7: 59-67.

16. L'Abbate A (1991). Pathophysiological basis for noninvasive functional evaluation of coronary stenosis. Circulation, 83 (Suppl III): III.2-III.7.

17. Lambertz $\mathrm{H}$, Kreis $\mathrm{A}$, Trumper $\mathrm{H} \&$ Hanrath $P$ (1990). Simultaneous transesophageal atrial pacing and transesophageal two-dimensional echocardiography: a new method of stress echocardiography. J ournal of the American College of Cardiology, 16: 1143-1153.

18. Michael TA, Antonescu A, Bhambi B \& Balasingam S (1996). Accuracy and usefulness of atrial pacing in conjunction with transthoracic echocardiography in the detection of cardiac ischemia. American J ournal of Cardiology, 77: 187-190.

19. Kamp O, De Cock CC, Kupper SJ, Roos J P \& Visser CA (1992). Simultaneous transesophageal two-dimensional echocardiography and atrial pacing for detecting coronary artery disease. American J ournal of Cardiology, 69: 1412-1416.

20. Popp R, Agaston A, Armstrong W, Nanda N, Pearlman A, Rakowski H, Seward J, Silverman N, Smith M, Stewart W, Taylor R, Thys D \& Davis C (1998). American Society of Echocardiography position papers. J ournal of the American Society of Echocardiography, 11: 95-98.

21. American Heart Association (1985). Position of the American Heart Association on research animal use: a statement for health professionals by a task force appointed by the board of directors of the American Heart Association. Circulation, 71: 849-850.

22. Babic R, Ostojic M, Stankovic G, Belesin B, Stojkovic S, Nedeljkovic M, PantelicBabic J \& Djordjevic-Dikic A (1994). Quantitative coronary arteriography versus stress echocardiography: Which is the "best" angiographic cut-off? J ournal of the American College of Cardiology, 38A (Abstract).

23. Agrawal $\mathrm{R}$, Schröder $\mathrm{K} \&$ Dingerkus $\mathrm{H}$ (1993). Must hyperdynamic response be considered when evaluating dobutamine 
stress echocardiography? Circulation, 88 (Suppl I): I-403 (Abstract).

24. Ofili EO, St Vrain JA \& Standeven JA (1993). Dobutamine stress echocardiography: Hemodynamic correlates and physiologic basis of wall thickening abnormality. J ournal of the American College of Cardiology, 21: 175A (Abstract).

25. Fung AY, Gallagher KP \& Buda AJ (1987). The physiologic basis of dobutamine as compared with dipyridamole stress interventions in the assessment of critical coronary stenosis. Circulation, 76: 943-951.

26. Picano E, Lattanzi F, Masini M , Distante A \& L'Abbate A (1986). High dose dipyridamole echocardiography test in effort angina pectoris. J ournal of the American College of Cardiology, 8: 846-854.

27. Segar DS, Ryan T, Sawada SG, J ohnson M \& Feigenbaum H (1995). Pharmacologically induced myocardial ischemia: a comparison of dobutamine and dipyridamole. J ournal of the American Society of Echocardiography, 8: 9-14.

28. Previtali $M$, Lanzarini $L$, Ferrario $M$, Tortorici M, Mussini A \& Montemartini C (1991). Dobutamine versus dipyridamole echocardiography in coronary artery disease. Circulation, 83 (Suppl III): III.27III.31.
29. Dagianti A, Penco M, Agati L, Sciomer S, Dagianti A, Rosanio S \& Fedele F (1995). Stress echocardiography: comparison of exercise, dipyridamole and dobutamine in detecting the extent of coronary artery disease. J ournal of the American College of Cardiology, 26: 18-25.

30. Wilkenshoff UM, Schröder $K$, Völler $H$, Dissman R, Dingerkus $H$, Linderer $T$ \& Schröder R (1994). Comparison of dobutamine and dipyridamole stress echocardiography in patients with single and multivessel disease undergoing PTCA. J ournal of the American College of Cardiology, 144A (Abstract).

31. Lanzarini L, Fetiveau R, Poli A, Mussini A \& Previtali M (1993). Comparison of dobutamine and dipyridamole stress-echo with exercise test for coronary artery disease diagnosis. J ournal of the American College of Cardiology, 21: 89A (Abstract).

32. Martin TW, Seaworth J F, J ohns J P, Pupa LE \& Condos WR (1992). Comparison of adenosine, dipyridamole and dobutamine in stress echocardiography. Annals of Internal Medicine, 116: 190-196.

33. Belesin BD, Ostojic M, Stepanovic J, Djordjevic-Dikic A, Stojkovic S, Nedeljkovic M, Stankovic G, Petrasinovic Z, Gojkovic L \& Vasiljevic-Pokrajcic Z (1994).
Stress echocardiography in the detection of myocardial ischemia. Head-to-head comparison of exercise, dobutamine and dipyridamole tests. Circulation, 90: 11681176.

34. Salustri A, Fioretti PM, McNeill AJ , Pozzoli MM \& Roetlandt J R (1992). Pharmacological stress echocardiography in the diagnosis of coronary artery disease and myocardial ischaemia: a comparison between dobutamine and dipyridamole. European Heart J ournal, 13: 1356-1362.

35. Paulsen PR, Pavek T, Crampton M, Bache RJ , Boudreau R \& Homans DC (1993). Which stress is best? Exercise, dobutamine, dipyridamole and pacing in an animal model. J ournal of the American College of Cardiology, 21: 90A (Abstract).

36. White CW, Wright CB \& Doty DB (1984). Does visual interpretation of the coronary arteriogram predict the physiologic importance of a coronary stenosis? New England J ournal of Medicine, 310: 819-824.

37. Gould KL \& Lipscomb K (1974). Effects of coronary stenoses on coronary flow reserve and resistance. American J ournal of Cardiology, 34: 48-54.

38. Picano E (1992). Stress Echocardiography. Springer Verlag, Berlin, Heidelberg. 